\title{
Sensitivity to auditory spectral width in the fetus and infant - an fMEG study
}

\section{Jana Muenssinger ${ }^{1}{ }^{*}$, Tamara Matuz $^{1,2+}$, Franziska Schleger ${ }^{1}$, Rossitza Draganova ${ }^{3}$, Magdalene Weiss $^{1,4}$, Isabelle Kiefer-Schmidt ${ }^{4}$, Annette Wacker-Gussmann ${ }^{5}$, Rathinaswamy B. Govindan ${ }^{6}$, Curtis L. Lowery ${ }^{7}$, Hari Eswaran ${ }^{7}$ and Hubert Preiss| ${ }^{1,2}$}

1 fMEG Center, University of Tuebingen, Tuebingen, Germany

2 Institute for Medical Psychology and Behavioral Neurobiology, University of Tuebingen, Tuebingen, Germany

${ }^{3}$ Department of Phoniatrics and Pediatric Audiology, Clinic of Otorhinolaryngology, Head and Neck Surgery, St. Elisabeth-Hospital Bochum, Bochum, Germany

${ }^{4}$ Department of Obstetrics and Gynecology, University Hospital Tuebingen, Tuebingen, Germany

${ }^{5}$ Department of Neonatology, University Children's Hospital Tuebingen, Tuebingen, Germany

${ }^{6}$ Division of Fetal and Transitional Medicine, Children's Hospital, Washington, DC, USA

${ }^{7}$ Department of Obstetrics and Gynecology, University of Arkansas for Medical Sciences, Little Rock, AR, USA

\section{Edited by:}

Christos Papadelis, Harvard Medical

School, USA

Reviewed by:

Minna Huotilainen, University of

Helsinki, Finland

Silvia Comani, Università degli Studi

"G. d'Annunzio," Italy

*Correspondence:

Jana Muenssinger, fMEG Center, University of Tuebingen,

Otfried-Müller-Strasse 47, Tuebingen 72076, Germany

e-mail: jana.muenssinger@

uni-tuebingen.de

' Jana Muenssinger and Tamara Matuz have contributed equally to this work.
Auditory change detection is crucial for the development of the auditory system and a prerequisite for language development. In neonates, stimuli with broad spectral width like white noise (WN) elicit the highest response compared to pure tone and combined tone stimuli. In the current study we addressed for the first time the question how fetuses react to "WN" stimulation. Twenty-five fetuses ( $M_{\text {age }}=34.59$ weeks $G A, S D \pm 2.35$ ) and 28 healthy neonates and infants ( $M_{\text {age }}=37.18$ days, $S D \pm 15.52$ ) were tested with the first paradigm, wherein $500 \mathrm{~Hz}$ tones, $750 \mathrm{~Hz}$ tones, and WN segments were randomly presented and auditory evoked responses (AERs) were measured using fetal magnetoencephalography ( $f M E G)$. In the second paradigm, 12 fetuses ( $M_{\text {age }}=25.7$ weeks $G A, S D \pm 2.4$ ) and 6 healthy neonates ( $M_{\text {age }}=23$ days and $S D \pm 6.2$ ) were presented with two auditory oddball conditions: condition 1 consisted of attenuated $W N$ as standard and $500 \mathrm{~Hz}$ tones and WN as deviants. In condition 2, standard $500 \mathrm{~Hz}$ tones were intermixed with WN and attenuated WN. AERs to volume change and change in spectral width were evaluated. In both paradigms, significantly higher AER amplitudes to WN than to pure tones replicated prior findings in neonates and infants. In fetuses, no significant differences were found between the auditory evoked response amplitudes of WN segments and pure tones (both paradigms). A trend toward significance was reached when comparing the auditory evoked response amplitudes elicited by attenuated WN with those elicited by WN (loudness change, second paradigm). As expected, we observed high sensibility to spectral width in newborns and infants. However, in the group of fetuses, no sensibility to spectral width was observed. This negative finding may be caused by different attenuation levels of the maternal tissue for different frequency components.

Keywords: WN, spectral width, auditory change detection, magnetoencephalography, auditory evoked responses

\section{INTRODUCTION}

Auditory change detection is an important prerequisite for a functional auditory system as well as for the development of language perception and can serve as an indicator for healthy cognitive functioning and development. It has been repeatedly shown that humans possess already at birth the capacity to process acoustic regularities and react to violations of such regularities, meaning that they are able to detect and discriminate different patterns of sound (Carral et al., 2005). Prior studies showed atypical auditory evoked responses (AERs) to auditory changes in pre-maturely born infants (Fellman et al., 2004) as well as in infants with genetic risk for dyslexia (Leppänen et al., 1999). However, the neurophysiological mechanisms underlying early sound perception and discrimination within both, typical and untypical development, are still not fully understood.
Most studies investigating change detection used oddball paradigms and evaluated AERs elicited by individual stimuli and mismatch negativity (MMN) responses determined by the difference between AERs elicited by standard and deviant stimuli. AERs are neurophysiologic indices of sensory functioning and their different components reflect basic cognitive functions. MMN (Näätänen, 2001) - a negative component which in adults peaks at around $150 \mathrm{~ms}$ after change onset - is considered as an indicator of automatic change detection reflecting discriminatory capacity. Assessing healthy infants, several studies showed that they were able to distinguish between pure tones of different frequencies (Kushnerenko et al., 2002; Huotilainen et al., 2003; Draganova et al., 2005, 2007). Kushnerenko et al. (2002) studied the development of change detection in neonates over the first 12 months and did not find statistically significant differences between the 
responses at different ages on the group level. They also showed that changes between pure tones and novel sounds (clicks, chirps, vowels, syllables) at the age of 2 years elicited significantly higher AER responses to novel sounds compared to pure tones. This effect might be explained by the "novelty" of the stimulus itself or could be caused by the change in spectral width between stimuli, because the novel sounds were composed of different frequencies. To test both hypotheses, Kushnerenko et al. (2007) investigated responses to pure tones, novel sounds, and white noise (WN) segments. WN segments were chosen to match the novel sounds in the broad frequency range but not in the novelty. Results revealed the highest responses for $\mathrm{WN}$ segments. This indicates that - unlike adults - neonates are highly sensitive to the effective amount of stimulation, with broader widths eliciting higher AERs. This finding is also supported by behavioral data showing that infants are more sensitive to the amount of stimulation than to the modality of stimulation (Lewkowicz and Turkewitz, 1980; Turkewitz et al., 1983).

However, as shown in several studies using fetal magnetoencephalography, auditory change detection occurs already in the fetal stage (Draganova et al., 2005, 2007; Huotilainen et al., 2005). MMN responses were observed as early as 28 weeks of gestation. In these studies, pure tones with a frequency of $500 \mathrm{~Hz}$ were used as standard stimuli, which were intermixed with rarely presented deviants with a frequency of $750 \mathrm{~Hz}$. However, to our best knowledge, fetal responses to more complex stimuli have not been investigated so far. One potential difficulty in using more complex stimuli in fetuses is the sound attenuation caused by maternal tissue. In general, it is known that high frequencies are more attenuated than lower frequencies [for review see Hepper and Shahidullah (1994)]. When using complex stimuli composed of multiple frequencies, the broad difference in attenuation levels between frequencies might result in the fact that fetuses perceive only parts of the whole frequency spectrum. This involves a decrement in the perceived differences between pure tones and complex stimuli by the fetus.

However, studies investigating fetal learning support the assumption that a broad spectrum of sounds reach the fetus in utero. Starting at the 29th week of gestational age, music as well as speech sounds were repeatedly presented to fetuses (Partanen et al., 2013a,b). Electroencephalographic (EEG) measurements directly after birth as well as 4 months after birth (Partanen et al., 2013a) showed enhanced AERs to the "learned" stimulus. AERs were significantly higher than in a control group of age-matched infants who were not presented with specific auditory stimulation before birth. This indicates that fetuses are able to perceive and learn a wide range of auditory stimuli in utero.

The aim of the first part of the current study (performed in Tuebingen) was to use a simplified paradigm to replicate the findings of Kushnerenko et al. (2007) in neonates and infants and to evaluate if the human fetal brain is able to process and react to differences in spectral width. Similar to newborns and infants, it was expected that fetuses will be sensitive to stimulus energy and will show increased AER amplitudes in reaction to the high spectral width of WN segments compared to those elicited by pure tones. In the second part of the study (performed in Little Rock), a within group oddball paradigm including both changes in volume and spectral width was used to further explore fetal cortical responses to different auditory changes. Higher AERs were expected for changes in frequency than for changes in volume.

\section{MATERIALS AND METHODS DATA ACQUISITION}

Data was recorded using SQUID Array for Reproductive Assessment (SARA) systems installed in two different locations, one at the Department of Obstetrics and Gynecology, University of Arkansas for Medical Sciences, AR, USA and a second one at the fMEG Center, University of Tuebingen. Both systems were built by VSM, Medical Technology Ltd., Canada. To avoid magnetic influences from the environment, the systems are installed in shielded rooms (Vakuumschmelze, Germany). The system in Little Rock contains 151 primary sensors and the system in Tuebingen 156 primary sensors. The primary sensors measure the biomagnetic signals from the physiological sources. Little Rock data were recorded with a sampling rate of $312.5 \mathrm{~Hz}$ and the Tuebingen data with $610.352 \mathrm{~Hz}$. The sensor arrangement in both systems is designed to fit the shape of the pregnant women's abdomen. Before each fetal measurement, an ultrasound is performed to determine fetal head position. Afterward, the mothers are seated on the fMEG device in the shielded room. Prior to the recording localization coils are placed at the left side, the right side and the spine of the mother as well as at the position on the abdomen where the fetal head is located. These coils indicate the maternal as well as the fetal position relative to the sensor array which are used in later data analysis.

\section{PARTICIPANTS FROM TUEBINGEN STUDY (FIRST PART)}

Twenty-five healthy pregnant women with uncomplicated pregnancies and normally developing fetuses $\left(M_{\mathrm{age}}=34.59\right.$ weeks, $\mathrm{SD} \pm 2.35$ weeks) between the gestational age of 30 and 38 weeks and 28 healthy newborns and infants without complications during pregnancy and birth between the age of 14 and 67 days $\left(M_{\text {age }}=37.18\right.$ days $\left.\mathrm{SD} \pm 15.52\right)$ participated in the study. The local ethical committee approved the study and a written consent was obtained.

\section{PROTOCOL USED IN TUEBINGEN STUDY (FIRST PART)}

For neonatal/infant measurements, a cradle was attached to the fMEG devise and neonates/infants were lying on their right side with their right hemisphere over the sensor array. Auditory stimulation was generated outside the shielded room and transmitted through flexible, air-filled tubes into the shielded room. Stimulation was presented with a sound pressure level of $65 \mathrm{~dB}$ to the left ear using earphones especially developed for neonatal measurements (bio-logic, USA). Stimulation consisted of the random presentation of a $500 \mathrm{~Hz}$ tone $(n=70)$, a $750 \mathrm{~Hz}$ tone $(n=70)$, and WN $(n=70)$. The stimulus duration was $0.5 \mathrm{~s}$ for the $500 \mathrm{~Hz}$ tone and the $750 \mathrm{~Hz}$ tone and $1 \mathrm{~s}$ for the "WN" $(80-1000 \mathrm{~Hz})$ segments. The inter-stimulus interval (ISI) between stimulus presentations varied randomly between 2 and $2.5 \mathrm{~s}$. The pure stimulation duration was $8 \mathrm{~min}$ (Figure 1).

All neonates/infants were measured while sleeping or lying quietly. During the whole measurement, one parent was inside the shielded room with the baby. 


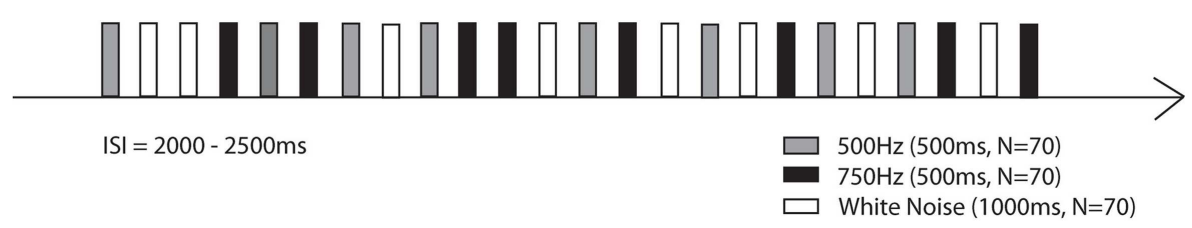

FIGURE 1 | Stimulation paradigm of the first part of the study (Tuebingen)

For the fetal measurements, the same paradigm was applied. Stimulation was presented using an air-filled balloon, which was placed directly on the maternal abdomen. Stimulation intensity was $95 \mathrm{~dB}$, which is attenuated by maternal tissue and amniotic fluid and which the fetus may perceive at a level of approximately 65-70 dB (Querleu et al., 1988).

\section{PARTICIPANTS FROM LITTLE ROCK STUDY (SECOND PART)}

Twelve healthy pregnant women with uncomplicated pregnancies and normally developing fetuses $\left(M_{\text {age }}=25.7\right.$ weeks GA, $\mathrm{SD} \pm 2.4)$ and six healthy newborns and infants without complications during pregnancy and birth $\left(M_{\text {age }}=23\right.$ days and $\left.\mathrm{SD} \pm 6.2\right)$ participated in the study. The local ethical committee approved the study and a written consent was obtained.

\section{PROTOCOL USED IN LITTLE ROCK STUDY (SECOND PART)}

Auditory stimulation was generated outside the shielded room and the stimuli were delivered to the inside of the shielded room through Tygon tubing that ended with an air-filled bag. Similar to the first paradigm, for the neonatal measurements a cradle was attached to the fMEG device and neonates/infants were lying on their side with one hemisphere over the sensor array. Stimulation was presented with a sound pressure level of $65 \mathrm{~dB}$ at the end of the acoustic emitter, which was attached to the ceiling at a distance of approximately $75 \mathrm{~cm}$ from the newborn's head. The stimulation paradigm consisted of two oddball conditions in which a $500 \mathrm{~Hz}$ tone, attenuated $\mathrm{WN}$ and $\mathrm{WN}$ were either standards (probability of 0.8 , presented for 560 times) or deviant sounds (each type with a probability of 0.1 , presented for 70 times) (see Figure 2). Total number of presented stimuli was 700 . The stimulus duration was $0.1 \mathrm{~s}$. The ISI was $1 \mathrm{~s}$. The total stimulation duration was about 17 min per condition. All neonates were measured while sleeping or lying quietly. During the measurement, one parent was inside the shielded room with the baby.

For fetal measurements, the same paradigm was used. Sound intensity measured at the end of the tube in the air was $115 \mathrm{~dB}$ for the $500 \mathrm{~Hz}$ tones as well as for the WN segments. For the attenuated WN segments the sound intensity at the end of the tube was $95 \mathrm{~dB}$.

\section{DATA ANALYSIS}

All fMEG recordings were filtered offline using a bandpass filter between 1 and $10 \mathrm{~Hz}$ for fetal recordings and between 1 and $15 \mathrm{~Hz}$ for neonatal recordings using Butterworth filter with zero phase distortion. Interfering maternal and fetal cardiac signals were attenuated using an orthogonal signal space projection technique
(Vrba et al., 2004; McCubbin et al., 2006) or a Hilbert transform method (Wilson et al., 2008). Data was cut in segments according to the test stimulus; the length of the segments was $200 \mathrm{~ms}$ before the stimulus (baseline) and $800 \mathrm{~ms}$ after the stimulus. All segments including artifacts due to maternal or fetal movement or muscle contractions higher than $2 \mathrm{pT}$ were excluded from further analysis. Evoked responses were determined by visual inspection and quantified by calculating the root-mean squares (RMS) of the five channels with the highest amplitudes. These RMS were used for statistical analysis.

\section{STATISTICAL ANALYSIS}

For the Tuebingen Study, the RMSs of all conditions were tested for normal distribution. For normally distributed data, a repeatedmeasures ANOVA with "tones" as within-subject factor was used to test for main effects and paired $t$-tests were used for post hoc comparisons. For non-normally distributed data, the non-parametric Friedman test was used to test for main effects. Significance levels were adjusted for multiple comparisons using Bonferroni correction.

Due to the explorative nature and the small sample size of the Little Rock study, the statistical analysis of this part had to be limited to descriptive indices and non-parametric comparative tests.

\section{RESULTS}

\section{RESULTS FROM TUEBINGEN STUDY (FIRST PART)} Neonatal/infant recordings

Fifteen neonates/infants were excluded from further analysis because either no measurement was possible due to movement/crying or because measurements had to be aborted due to agitation of the neonate/infant. Statistical analysis has been done on the remaining 13 datasets $\left(M_{\text {age }}=34.08\right.$ days, $\left.\mathrm{SD} \pm 15.18\right)$.

A significant main effect of tones was observed $(F=11.45$, $p<0.05)$. Post hoc analysis revealed significant differences between the $500 \mathrm{~Hz}$ tone and $\mathrm{WN}\left(T_{12}=-3.82, p<0.0167\right)$ and the $750 \mathrm{~Hz}$ tone and $\mathrm{WN}\left(T_{12}=-3.22, p<0.0167\right)$. No significant differences between the $500 \mathrm{~Hz}$ tone and the $750 \mathrm{~Hz}$ tone were observed $\left(T_{12}=-1.05, p=0.317\right.$, see Figure 3$)$.

\section{Fetal recordings}

Eleven fetuses were excluded from further analysis because they did not show visible evoked responses for all three conditions. All further analysis is based on the remaining 14 fetuses $\left(M_{\text {age }}=34.57, \mathrm{SD} \leq 2.34\right)$.

No significant main effect of tones was found for fetal recordings $\left(\chi^{2}=2.71, p=0.26\right.$, see Figure 4$)$. 


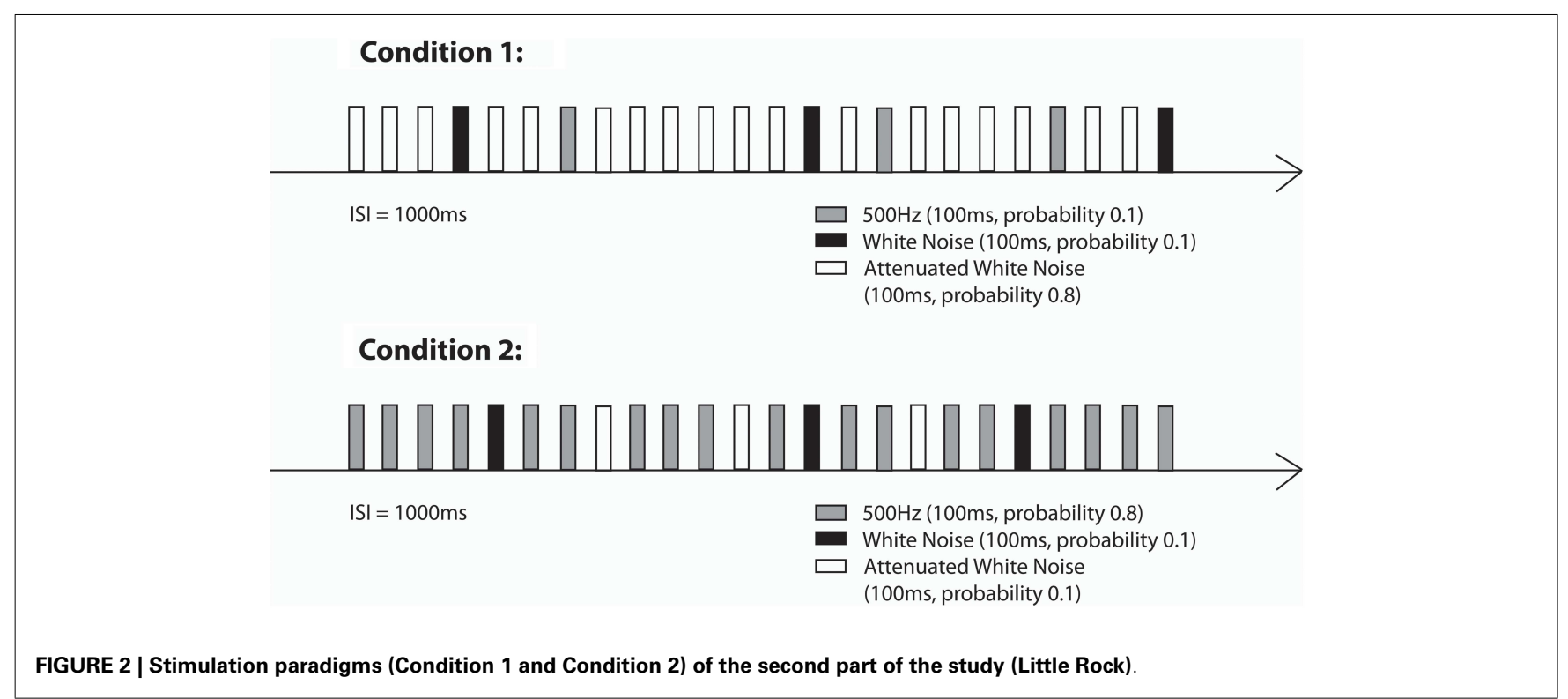

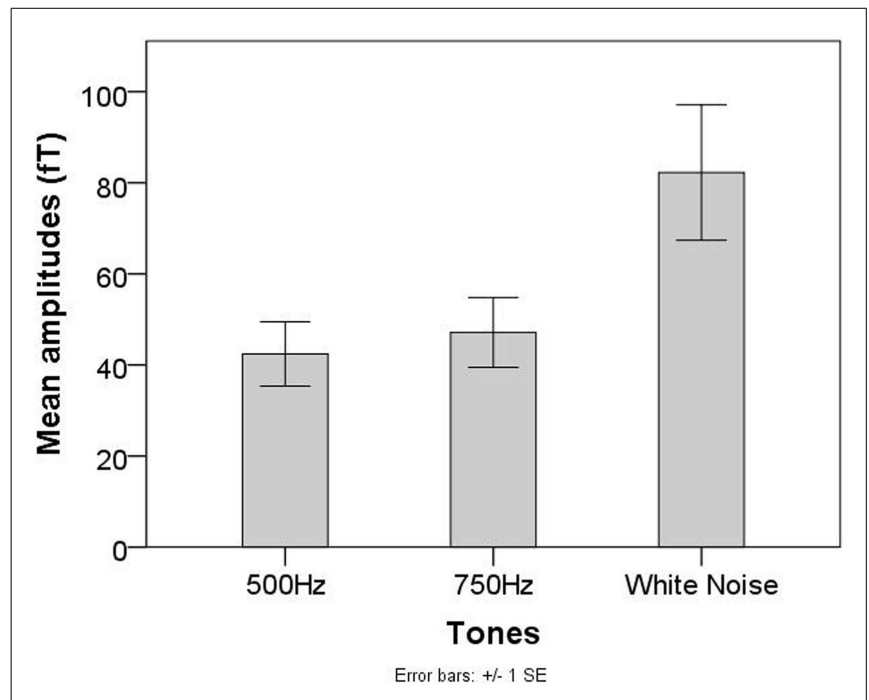

FIGURE 3 | Mean AER amplitudes for the $500 \mathrm{~Hz}, 750 \mathrm{~Hz}$, and WN stimuli in the group of neonates/infants of the Tuebingen study.

\section{RESULTS FROM LITTLE ROCK STUDY (SECOND PART) Neonatal/infant recordings}

One out of six neonatal/infant recordings was excluded from the analysis because it was stopped within the first 5 min of the measurement due to newborn crying. The RMS values of the amplitudes of the AERs for the neonates are shown in Table 1.

Mean RMS value for the WN segments in the first oddball condition $(M=37.7, \mathrm{SD} \pm 5.6)$ was higher than the mean of the RMS values for the $500 \mathrm{~Hz}$ tones $(M=28.7, \mathrm{SD} \pm 9.2)$. The same was true for the second condition between the amplitudes of the WN segments $(M=40.6, \mathrm{SD} \pm 18.3)$ and those of the attenuated $\mathrm{WN}$ segments $(M=27.8, \mathrm{SD} \pm 0.04)$. Wilcoxon signed ranked tests showed significant differences $(Z=3.7, p<0.05)$ between

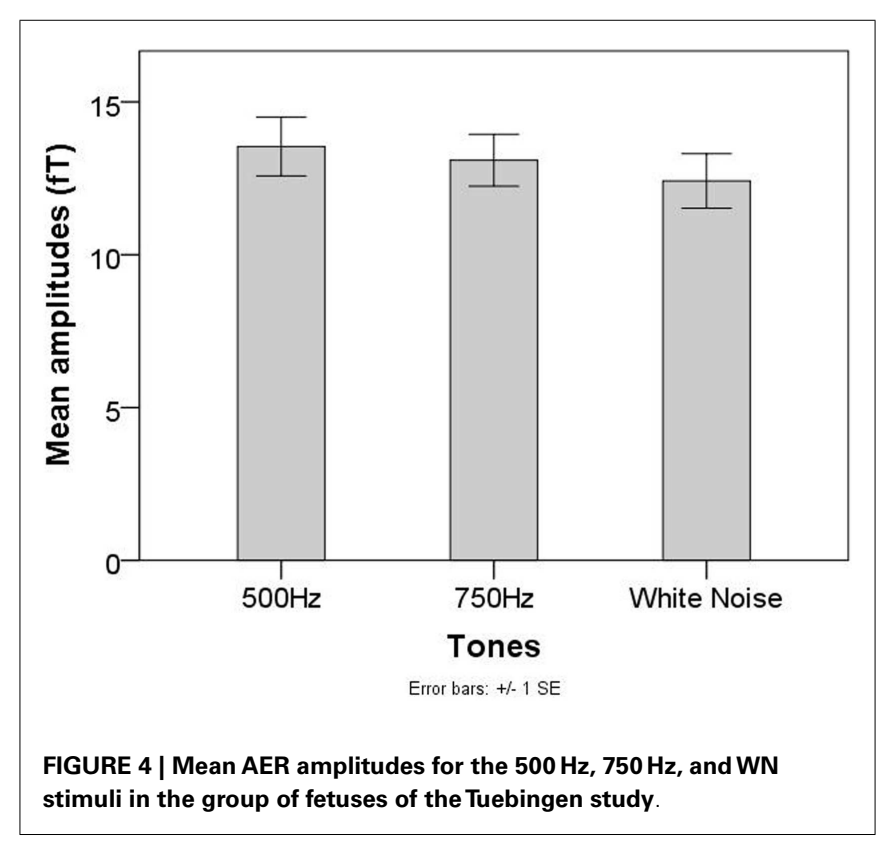

the median amplitudes of the AERs to the $\mathrm{WN}$ and those to the attenuated $\mathrm{WN}$ segments as well as between the amplitudes of the cortical responses to the $\mathrm{WN}$ and those to the $500 \mathrm{~Hz}$ tones of the second condition $(Z=2.02, p<0.05)$.

\section{Fetal recordings}

Two out of 12 fetal recordings were excluded from further analysis based on maternal movements and large maternal and fetal heart residuals. AERs on the deviants were detected in 8 out of 10 fetuses in the first oddball condition and in 5 out of 10 fetuses in the second one. Amplitudes of the AERs elicited by infrequent WN segments were not significantly different than those elicited by the infrequent $500 \mathrm{~Hz}$ tones $(Z=-0.70, p=0.48)$. The difference between RMS values of AERs elicited by infrequent WN segments 
Table 1 | RMS values of the neonatal AERs for both oddball conditions applied in the second stimulation paradigm.

\begin{tabular}{lccccc}
\hline Subject & \multicolumn{2}{c}{ First oddball condition } & & \multicolumn{2}{c}{ Second oddball condition } \\
\cline { 2 - 3 } \cline { 5 - 6 } RMS & WN (fT) & $\mathbf{5 0 0 ~ H z ~ ( f T ) ~}$ & & WN (fT) & WN attenuated (fT) \\
\hline WN01 & 30.70 & 14.99 & & 17.90 & 11.49 \\
WN02 & 33.50 & 27.30 & & 30.70 & 26.40 \\
WN03 & 42.20 & 29.20 & & 67.10 & 29.32 \\
WN04 & 38.00 & 31.10 & & 42.30 & 35.68 \\
WN05 & 44.00 & 40.70 & & 45.00 & 36.20 \\
\hline
\end{tabular}

and those elicited by infrequent attenuated $\mathrm{WN}$ segments showed a tendency toward significance $(Z=-1.8, p=0.08)$.

\section{DISCUSSION}

The current study aimed to use a simplified paradigm to replicate the increased neonatal reaction to $\mathrm{WN}$ stimuli which was already found by Kushnerenko et al. (2007). Moreover, WN segments were used for the first time in fetal recordings to investigate if the human brain is able to detect changes in the spectral width of auditory stimulation during fetal period. An oddball paradigm including changes in volume and spectral width was used to further investigate fetal change detection.

Results of the first paradigm showed, that even when using a simple paradigm wherein pure tones and WN segments were presented in random order with the same probability, infants showed significantly higher reactions to the $\mathrm{WN}$ segments than to the pure tones. This replicates prior results showing that infants are sensitive to the effective amount of stimulation with higher reactions to high-energy stimulation (in this case high spectral width) and lower reactions to low-energy stimulation (Kushnerenko et al., 2007). Also earlier behavioral studies support these findings (Lewkowicz and Turkewitz, 1980). Even though our results are in line with other studies, it has to be taken into account that the duration of the $\mathrm{WN}$ segments was longer than that of pure tones in the current study. Therefore, the higher reaction to the WN segments might partly be explained by the change in duration rather than in spectral width. However, when studying change detection using a multiple deviant paradigm, Sambeth et al. (2009) found no significant differences between amplitudes of the reaction to standards and duration deviants using magnetoencephalography. This indicates that a change in tone duration plays only a minor role concerning the increment of AER amplitudes. Our results from the second paradigm further support this finding. Neonatal/infant cortical responses to WN segments showed significantly higher amplitudes when compared to pure tones and attenuated WN segments of the same duration. Nevertheless, additional data is needed to thoroughly address this question.

Different from neonates and infants, in both fetal paradigms stimuli consisting of multiple frequencies did not elicit AERs with higher amplitudes than pure tones. Special requirements for fetal measurements might be one important factor concerning the evaluated group differences between neonates and fetuses. While neonates can be stimulated directly at the ear with sounds transmitted through the air, fetal stimulation takes place outside the maternal abdomen. Since the fetus is surrounded by amniotic fluid, the characteristics of sound transmission are different than sound transmission in air. Different studies have been performed to evaluate sound attenuation in fetal measurements [Hepper and Shahidullah (1994) and references therein]. Using a hydrophone near the fetal head at the beginning of labor and after amniotomy, Querleu et al. (1988) recorded an attenuation of $2 \mathrm{~dB}$ at $250 \mathrm{~Hz}$, $14 \mathrm{~dB}$ at $500 \mathrm{~Hz}, 20 \mathrm{~dB}$ at $1000 \mathrm{~Hz}$, and $26 \mathrm{~dB}$ at $2000 \mathrm{~Hz}$. Generally, higher frequencies are more attenuated than lower frequencies, which in some works are even reported to be slightly enhanced in volume (Richards et al., 1992). Since the WN segments in the current study were composed of frequencies in the range between 50 and $1000 \mathrm{~Hz}$, which were all presented with the same sound pressure level, we assume that the higher frequencies were attenuated much more than the lower frequencies before they reached the fetal ear. Compared to neonates/infants who heard the full spectral width of WN segments, fetuses might have perceived only parts of the frequency range, narrowed down to lower and middle frequencies. In this case, due to a narrowed perceived spectral width, also the total energy of the stimulation would have been weakened, leading to AER amplitudes comparable with those of pure tones. Additionally to the differences in sound transmission between fetuses and neonates, the background noise differs between those groups. While neonates are stimulated in a quiet environment, fetuses are surrounded by background noises caused by the maternal heart beat, bowel movements or the maternal voice. Different studies reported background noise intensities of $72 \mathrm{~dB}$ measured during labor in the uterus (Bench, 1968), $85 \mathrm{~dB}$ measured before labor, and even $95 \mathrm{~dB}$ after the R-wave of the maternal heart (Walker et al., 1971). Since these background noises are of low frequencies, we believe that they might have superimposed and masked the lower frequency ranges of WN segments, leaving the higher frequencies nearly unaffected. Since the low frequencies are less attenuated but covered by the background noise, the perceived frequency range is additionally narrowed down. Even though learning studies in fetuses (Partanen et al., 2013a,b) clearly showed that fetuses are able to perceive and learn melodies and speech sounds, these studies did not focus on the effective amount of stimulation. Even if a high range of stimuli can be perceived when presented separately, no clear conclusion can be drawn concerning the frequencies perceived by the fetus when a broad range of frequencies are presented at the same time with the same intensity. Further research is needed to entirely solve this question.

Taken together, we assume that fetuses probably did not perceive the full frequency range of the $\mathrm{WN}$ stimulus, which weakened stimulus energy and made it less distinguishable from the pure tone stimuli. The results of the second study suggest that the neonates are sensitive to both spectral and volume changes, whereas the fetuses are rather sensitive to loudness change.

It has to be stressed that the two studies were performed with two separate systems for fMEG recordings and the results were comparable, providing further support for the $\mathrm{AMEG}$ as a reliable method to assess fetal brain development.

In summary, the current study showed that newborns are able to differentiate between pure tones and $\mathrm{WN}$ segments even in simple paradigms, where tones are randomly presented with the same probability. This enables shorter measurement times, which is an important point in neonatal measurements since movement artifacts are highly probable especially in long measurements. To 
thoroughly evaluate fetal ability to distinguish between high- and low-energy stimulation, stimulation methods need to be developed which are not attenuated by the maternal abdomen and amniotic fluid and therefore ensure that the fetus receives the complete spectrum of the WN.

\section{AUTHORS CONTRIBUTION}

Jana Muenssinger was involved in the development of the design, data acquisition and analysis, statistical analysis, and wrote the manuscript. Tamara Matuz was involved in the development of the design, data acquisition and analysis, statistical analysis, and writing of the manuscript. Franziska Schleger was involved in data acquisition and analysis and revised the manuscript. Rossitza Draganova was involved in the data analysis and revised the manuscript. Magdalene Weiss was involved in the recruitment of subjects, data acquisition, and revised the manuscript. Isabelle Kiefer-Schmidt and Annette Wacker-Gussmann were involved in the recruitment of subjects and revised the manuscript. Rathinaswamy B. Govindan was involved in the development of the paradigm, data analysis, and revised the manuscript. Curtis L. Lowery was involved in the conceptual design of the study and revised the manuscript. Hari Eswaran was involved in designing the study, analyzing and interpreting results, and revised the manuscript. Hubert Preissl was involved in the development of the design, data analysis and revised the manuscript.

\section{ACKNOWLEDGMENTS}

We thank all families for their participation. The fetal MEG and the authors in Tuebingen were supported by the Deutsche Forschungsgemeinschaft (DFG BI 195-50), the Ministry of Science, BadenWürttemberg, the Werner Reichardt Center for Integrative Neuroscience (CIN, Pool-Project 2009-2013) and the Open Access Publishing Fund of Tuebingen University (provided by the "Deutsche Forschungsgemeinschaft"). The Little Rock study was partially supported by grants from the National Institutes of Health (NIH) R01EB007826 and 5R01NS036277-03, USA.

\section{REFERENCES}

Bench, J. (1968). Sound transmission to the human foetus through the maternal abdominal wall. J. Genet. Psychol. 113, 85-87. doi:10.1080/00221325.1968. 10533811

Carral, V., Huotilainen, M., Ruusuvirta, T., Fellman, V., Näätänen, R., and Escera, C. (2005). A kind of auditory 'primitive intelligence' already present at birth. Eur. J. Neurosci. 21, 3201-3204. doi:10.1111/j.1460-9568.2005.04144.x

Draganova, R., Eswaran, H., Murphy, P., Huotilainen, M., Lowery, C., and Preissl, H. (2005). Sound frequency change detection in fetuses and newborns, a magnetoencephalographic study. Neuroimage 28, 354-361. doi:10.1016/j.neuroimage. 2005.06.011

Draganova, R., Eswaran, H., Murphy, P., Lowery, C., and Preissl, H. (2007). Serial magnetoencephalographic study of fetal and newborn auditory discriminative evoked responses. Early Hum. Dev. 83, 199-207. doi:10.1016/j.earlhumdev.2006. 05.018

Fellman, V., Kushnerenko, E., Mikkola, K., Ceponiene, R., Leipälä, J., and Näätänen, R. (2004). Atypical auditory event-related potentials in preterm infants during the first year of life: a possible sign of cognitive dysfunction? Pediatr. Res. 56, 291-297. doi:10.1203/01.PDR.0000132750.97066.B9

Hepper, P. G., and Shahidullah, B. S. (1994). Development of fetal hearing. Arch. Dis. Child. 71, F81-F87. doi:10.1136/fn.71.2.F81

Huotilainen, M., Kujala, A., Hotakainen, M., Parkkonen, L., Taulu, S., Simola, J., et al. (2005). Short-term memory functions of the human fetus recorded with magnetoencephalography. Neuroreport 16, 81-84. doi:10.1097/00001756-20050119000019
Huotilainen, M., Kujala, A., Hotakainen, M., Shestakova, A., Kushnerenko, E., Parkkonen, L., et al. (2003). Auditory magnetic responses of healthy newborns. Neuroreport 14, 1871-1875. doi:10.1097/00001756-20031006000023

Kushnerenko, E., Ceponiene, R., Balan, P., Fellman, V., and Näätänen, R. (2002). Maturation of the auditory change detection response in infants: a longitudinal ERP study. Neuroreport 13, 1843-1848. doi:10.1097/00001756-20021028000002

Kushnerenko, E., Winkler, I., Horvath, J., Naatanen, R., Pavlov, I., Fellman, V., et al. (2007). Processing acoustic change and novelty in newborn infants. Eur. J. Neurosci. 26, 265-274. doi:10.1111/j.1460-9568.2007.05628.x

Leppänen, P., Pikho, E., Eklund, K. M., and Lyytinen, H. (1999). Cortical responses of infants with and without a genetic risk for dyslexia: II. Group effects. Neuroreport 10, 969-973. doi:10.1097/00001756-199904060-00014

Lewkowicz, D. J., and Turkewitz, G. (1980). Cross-modal equivalence in early infancy: auditory - visual intensity matching. Dev. Psychol. 16, 597-607. doi:10.1037/0012-1649.16.6.597

McCubbin, J., Robinson, S. E., Cropp, R., Moiseev, A., Vrba, J., Murphy, P., et al. (2006). Optimal reduction of MCG in fetal MEG recordings. IEEE Trans. Biomed. Eng. 53, 1720-1724. doi:10.1109/TBME.2006.876619

Näätänen, R. (2001). The perception of speech sounds by the human brain as reflected by the mismatch negativity (MMN) and its magnetic equivalent (MMNm). Psychophysiology 38, 1-21. doi:10.1111/1469-8986.3810001

Partanen, E., Kujala, A., Tervaniemi, M., and Huotilainen, M. (2013a). Prenatal music exposure induces long-term neural effects. PLoS ONE 8:e78946. doi:10.1371/journal.pone.0078946

Partanen, E., Kujala, T., Näätänen, R., Liitola, A., Sambeth, A., and Huotilainen, M. (2013b). Learning-induced neural plasticity of speech processing before birth Proc. Natl. Acad. Sci. U.S.A. 110, 15145-15150. doi:10.1073/pnas.1302159110

Querleu, D., Renard, X., Versyp, F., Paris-Delrue, L., and Crepin, G. (1988). Fetal hearing. Eur. J. Obstet. Gynecol. Reprod. Biol. 28, 191-212. doi:10.1016/00282243(88)90030-5

Richards, D. S., Frentzen, B., Gerhardt, K. J., McCann, M. E., and Abrams, R. M. (1992). Sound Levels in the Human Uterus. Obstet. Gynecol. 80, 186-190.

Sambeth, A., Pakarinen, S., Ruohio, K., Fellman, V., Van Zuijen, T. L., and Huotilainen, M. (2009). Change detection in newborns using a multiple deviant paradigm: a study using magnetoencephalography. Neurophysiol. Clin. 120, 530-538. doi:10.1016/j.clinph.2008.12.033

Turkewitz, G., Lewkowicz, D. J., and Gardner, J. M. (1983). "Determinants of infant perception," in Advances in the Study of Behavior, eds C. Rosenblatt, C. Beer, R. Hinde, and M. Busnel (New York: Academic Press), 39-62.

Vrba, J., Robinson, S. E., McCubbin, J., Lowery, C. L., Eswaran, H., Wilson, J. D., et al. (2004). Fetal MEG redistribution by projection operators. IEEE Trans. Biomed. Eng. 51, 1207-1218. doi:10.1109/TBME.2004.827265

Walker, D., Grimwade, J., and Wood, C. (1971). Intrauterine noise: a component of the fetal environment. Am. J. Obstet. Gynecol. 109, 91-95.

Wilson, J. D., Govindan, R. B., Hatton, J. O., Lowery, C. L., and Preissl, H. (2008). Integrated approach for fetal QRS detection. IEEE Trans. Biomed. Eng. 55, 2190-2197. doi:10.1109/TBME.2008.923916

Conflict of Interest Statement: The authors declare that the research was conducted in the absence of any commercial or financial relationships that could be construed as a potential conflict of interest.

Received: 01 October 2013; paper pending published: 13 October 2013; accepted: 15 December 2013; published online: 31 December 2013.

Citation: Muenssinger J, Matuz T, Schleger F, Draganova R, Weiss M, Kiefer-Schmidt I, Wacker-Gussmann A, Govindan RB, Lowery CL, Eswaran H and Preissl H (2013) Sensitivity to auditory spectral width in the fetus and infant-an fMEG study. Front. Hum. Neurosci. 7:917. doi: 10.3389/fnhum.2013.00917

This article was submitted to the journal Frontiers in Human Neuroscience. Copyright (c) 2013 Muenssinger, Matuz, Schleger, Draganova, Weiss, Kiefer-Schmidt, Wacker-Gussmann, Govindan, Lowery, Eswaran and Preissl. This is an open-access article distributed under the terms of the Creative Commons Attribution License (CC $B Y)$. The use, distribution or reproduction in other forums is permitted, provided the original author(s) or licensor are credited and that the original publication in this journal is cited, in accordance with accepted academic practice. No use, distribution or reproduction is permitted which does not comply with these terms. 